\title{
SEROLOGICAL CHARACTERIZATION AND ANTIMICROBIAL SUSCEPTIBILITY PATTERNS OF CLINICAL ISOLATES OF SALMONELLA FROM PATIENTS ATTENDING GENERAL HOSPITAL, FUNTUA, NIGERIA
}

\author{
*Abdullahi, B. ${ }^{1}$, Olonitola, O. S. ${ }^{2}$, Jatau, E. D ${ }^{2}$. and Usman, A. D ${ }^{3}$. \\ ${ }^{1}$ Department of Bilogy, Umaru Musa Yar'adua University, Katsina. \\ ${ }^{2}$ Department of Microbiology, Ahmadu Bello Univeristy, Zaria. \\ ${ }^{3}$ Department of Biological Sciences, Bayero University, Kano. \\ *Correspondence author: albishir13@yahoo.com, 08054527359
}

\begin{abstract}
Serological characterization and antimicrobial susceptibility patterns of clinical isolates of Salmonella were carried out for a period of 8 -months to study the most frequently encountered serovars in salmonellosis and their antimicrobial susceptibility patterns. Two hundred and forty samples from both stool and blood specimens were collected from out patients attending General Hospital, Funtua, Katsina state of Nigeria. The samples were collected from patients diagnosed by clinicians of having either pyrexia, gastroenteritis or both. Samples were cultured, isolates identified and antibiotic susceptibility test was performed using standard procedures. The total number of 29(12.1\%) of the 240 samples collected were identified as Salmonella strains. Out of the 29 isolates, 19(65.5\%) were responsible for typhoidal salmonellosis while 10(34.5\%) were responsible for non-typhoidal salmonellosis. Of the 29 cases of salmonellosis, 24(82.8\%) were from children and 5(17.2.7\%) from adu/ts. However, the age of the patients have no significant relationship in both the typhoidal and non-typhoidal diseases, with their $p$ values $=0.109,0.784>$ 0.05 respectively. S. typhi 16(55.2\%) was the most frequent/y encountered, followed by $S$. enteritidis $7(24.1 \%)$ and $3(10.3 \%)$ each for $S$. paratyphi $A$ and $S$. typhimurium. Of the total isolates, 26(89.7\%) were found to be resistant to Ampicillin, 6(20.7\%) resistant to Cefotaxime, $24(82.8 \%)$ resistant to Chloramphenicol, 9(31\%) resistant to Co-trimoxazole and 2(6.9\%) resistant to Nalidixic acid. However, resistance to Ofloxacin and Ciprofloxacin by the isolates were not observed. There was no statistically significant difference $(p>0.05)$ in antimicrobial resistance patterns exhibited among typhoidal and non-typhoidal Salmonellae. Therefore, Fluoroquinolones are recommended as the drug of choice for both typhoidal and non-typhoidal salmonellosis, although, caution should be taken by the clinicians in prescribing them in order to avoid resistance to these drugs.

Keywords: Salmonella; occurrence; characterization; susceptibility; fluoroquinolones.
\end{abstract}

\section{INTRODUCTION}

Salmonella is a genus of rod-shaped, Gram-negative, non-spore forming, predominantly motile bacteria that obtain their energy from oxidation and reduction reactions using organic sources. They are facultative anaerobes and most species produce hydrogen sulphide (Giannella, 1996).

The bacterial genus, Salmonella is divided into two species, Salmonella bongori and $S$. enterica. $S$. enterica itself comprised of six subspecies viz; $S$. enterica subsp. enterica, $S$. enterica subsp. salamae, $S$. enterica subsp. arizonae, $S$. enterica subsp. diarizonae, $S$. enterica subsp. indica, and $S$. enterica subsp. houtenae, or I, II, IIIa, IIIb, IV, and VI, respectively (Porwollik et al., 2003). Of these six subspecies, only subspecies I is associated with disease in warm-blooded animals. To date, there exist over 2,300 serovars identified within subspecies I. However, only a small fraction of the thousands of described subspecies I serovars frequently cause disease in humans and domestic animals. For example, the annual report of the Centers for Disease Control and Prevention (CDC) registered 360 different serovars in human infections in the U.S. in 2009.
Approximately $50 \%$ of these infections were caused by only three Salmonella serovars, specifically $S$. typhimurium, $S$. enteritidis and $S$. newport. The 12 most prevalent Salmonella serovars were responsible for $>70 \%$ of all human Salmonella infections (McClelland et al., 2004).

Typhoidal salmonellosis and non-typhoidal salmonellosis are major health problems in developing countries in which Nigeria belongs. The problems, according to many findings are associated with unhygienic processing and preparation of foods, substandard water supply, inadequate sanitary measures and emergence of multidrug resistance in some Salmonella strains (Threlfall and Ward, 2001 and Wright et al., 2005). Contaminated food is the major source of transmission of salmonellosis (Guithrie, 1991). Frequency of occurrence is greatest in foods which are not cooked or have been incompletely cooked, such as roasted meat (in which Suya and Balangu belongs) or roasted chicken. Eating of half cooked Suya or Balangu processed at road side under unhygienic condition is a common practice in Nigeria, there by exposing the consumers to Salmonella infections (Wright et al, 2007). 
The collapse of primary healthcare system couple with the unavailability of drugs in hospitals in this country has resulted in most people resulting to purchasing drugs on counters and in some cases from road side sellers instead of going to the hospitals there by exposing them to the danger of acquiring multidrug resistant (MDR) microorganisms (Akinyemi et al, 2007).

S. typhi causes typhoid fever (enteric fevers) only in humans. Other serotypes, namely, non-typhoidal Salmonella serotypes, such as $S$. typhimurium, $S$. enteritidis and $S$. choleraesius can cause a wide spectrum of diseases in human and animals, such as acute gastroenteritis, bacteremia, and extraintestinally localised infection involving many organs (Lin-Hui et al., 2004). A syndrome similar to typhoid fever is caused by paratyphoidal serotypes of Salmonella. The paratyphoid serotypes (i.e., $S$. paratyphi A, $S$. paratyphi $\mathrm{B}$, and $S$. paratyphi $\mathrm{C}$ ) are isolated less frequently than $S$. typhi (Mindy, 2003).

Like other developing countries, treatment of patients in Nigeria has been based on the use of first line (conventional) antibiotics, such as ampicillin, chloramphenicol and co-trimoxazole and the third generation cephalosporins. However, efficacies of some of these drugs have been doubtful, following the emergence of multidrug resistance in Salmonella strains (Akinyemi et al., 2000). Flouroquinolones have been found to be efficacious both in vitro and in vivo in the treatment of several Salmonella-associated illnesses, although strains with reduced susceptibility to ciprofloxacin among travellers have been reported in some parts of the globe (Hakanen et al., 2001).

So far as we know there has been no reported data on characterization and distribution of Salmonella serotypes and their antimicrobial resistivity patterns from patients diagnosed to be suffering from Salmonella infection in General Hospital, Funtua. This form the basis for the serological characterization and antimicrobial susceptibility patterns of Salmonella isolates implicated in typhoidal and non-typhpoidal salmonellosis in General hospital, Funtua, Katsina state of Nigeria.

\section{MATERIALS AND METHODS}

The samples were collected from patients diagnosed by clinicians of having either pyrexia, gastroenteritis or both. Both blood and stool were collected from each patient. Three millimetre of blood and a loopful of fresh stool collected from each patient were directly inoculated into $27 \mathrm{ml}$ of trypticase soy broth (PRO-LAB Diagnostic, USA) and $9 \mathrm{ml}$ of selenite- $F$ broth (PROLAB Diagnostic, USA) contained in bijou bottle respectively. The tubes containing the samples were transported to Biological sciences Laboratory of Umaru Musa Yar'adua University, Katsina, and incubated for 24 hours at $37^{\circ} \mathrm{C}$.

\section{Isolation and identification of Salmonella spp. based on cultural and biochemical characteristics}

A loopful of the culture in tripticase soy broth and selenite-F broth were streaked seperately on Xylose
Lysine Deoxycholate (XLD) agar, Brilliant Green Agar (BGA), MacConkey (MC) agar, Desoxycholate Citrate (DC) agar and Salmonella-Shigella (SS) agar. Cultures were incubated for 24 hours at $37^{\circ} \mathrm{C}$, and colonies grown on the plates were selected for biochemical characterization as described by Barrow and Feltham (1993). The comercially-available identification system-API 20E (bio Mereux, France) was used.

\section{Serotyping of identified Salmonella species}

Colonies considered to be of Salmonella spp. were further tested for somatic (O) and flagella (H) antigens with polyvalent antisera (Serotype-Lab, Thailand).

\section{Antibiotic susceptibility testing}

Antibiotic susceptibility tests were carried out on the Salmonella serotypes. Seven antibiotics were screened using disc diffusion methods on Mueller-Hilton Agar. Three colonies were inoculated into a tube containing tryptic soy broth (PRO-LAB Diagnostic, USA ) and incubated at $37^{\circ} \mathrm{C}$. Standardization of the inocula was performed. The turbidity of the inocula was adjusted to match that of 0.5 McFarland standard. Within 15 minutes of preparing the adjusted inocula, a sterile cotton swab was dipped into the inocula. The swab was rotated several times and pressed firmly on the inside wall of the tube above the fluid level to remove excess inocula from the swab. The surface of the Mueller-Hinton Agar plate was incubated by running the swab around the rim of the Agar. Sterile forcep was used to dispense the single discs (PRO-LAB Diagnostic, USA) onto the Mueller-Hinton Agar surface. The standard discs used for susceptibility testing include ofloxacin $(20 \mu \mathrm{g})$, nalidixic acid $(30 \mu \mathrm{g})$, ciprofloxacin $(20 \mu \mathrm{g})$, co-trimoxazole $(25 \mu \mathrm{g})$, ampicillin $(25 \mu \mathrm{g})$, chloramphenicol $(30 \mu \mathrm{g})$ and third generation ceftazidime $(25 \mu)$. After 24 hours of incubation at $37^{\circ} \mathrm{C}$, zones of inhibition were measured and recorded with the help of vernier caliper in millimeters $(\mathrm{mm})$. The standard zones of inhibition interpretation chart by WHO (2010) was used to interpret the zonesof inhibition. Escherichia coli ATCC 25922 was used as control.

\section{RESULTS}

Out of the 240 samples of both blood and stool screened, 29 Salmonella were isolated, amountng to $12.1 \%$ incidence rate. Based on the symptoms manifestation and information extracted from the questionnaire, nineteen Salmonella isolates were implicated in typhoidal salmonellosis and ten were implicated in non-typhoidal salmonellosis. Therefore, of the 29 cases of salmonenosis, $65.5 \%$ typhoidal salmonellosis and $34.5 \%$ of non-typhoidal salmonellosis were recorded. Sixteen $S$. typhi were isolated, accounting for $55.2 \%$ of the total number of the isolates. Of the $16 S$. typhi isolates, 8(50\%) were recovered from blood only, $2(12.5 \%)$ from stool only while $6(37.5 \%)$ from both blood and stool of the patients. All the $3 S$. paratyphi A isolated were recovered from blood. Both $S$. enteritidis and $S$. typhimurium were recovered from stool only (Table.1). 
The $S$. typhi were recovered from patient belonging to age-groups $0-5,6-15,16-30$ and $\geq 46$ yrs. Three $(10.3 \%)$ S. paratyphi A were isolated and all were implicated in typhoidal salmonellosis. The $S$. paratyphi A were found among age-group 6-15yrs. Seven (24.1\%) S. enteritidis were isolated and all were implicated in non-typhoidal salmonellosis, distributed among age-groups 0-5 and 6-15yrs. Three (10.3\%) $S$. typhimurium were isolated and implicated in nontyphoidal salmonellosis which were isolated among age-group $0-5$ years. Twelve (41.4\%) of the total isolates were recovered from each of the age-group 0$5 y$ rs and $6-15 y r s$. Therefore, $82.8 \%$ of the total cases were associated with children while $17.2 \%$ cases were associated with adults (Table 2). Males accounted for $19(65.5 \%)$ cases of salmonellosis while females accounted for $10(34.5 \%)$ cases (Fig. 1). In this study, S. typhi and S. paratyphi A were $100 \%$ resistant to ampicillin, $93.8 \%$ and $66.7 \%$ respectively resistant to chloramphenicol while resistance to cotrimoxazole were $37.5 \%$ and $66.7 \%$ respectively. The resistance of both serotypes to fluoroquinolones (ciprofloacine and ofloxacin) was not observed. Resistances to Ampicillin by $S$. enteritidis and $S$. typhimurium (nontyphoidal salmonellae) were $57.1 \%$ and $100 \%$ respectively while that of both the non-typhoidal salmonellae to Fluoroquinolones was not observed (Figure 2).

Table 1. Cases of Salmonellosis and their Implicated Serotypes.

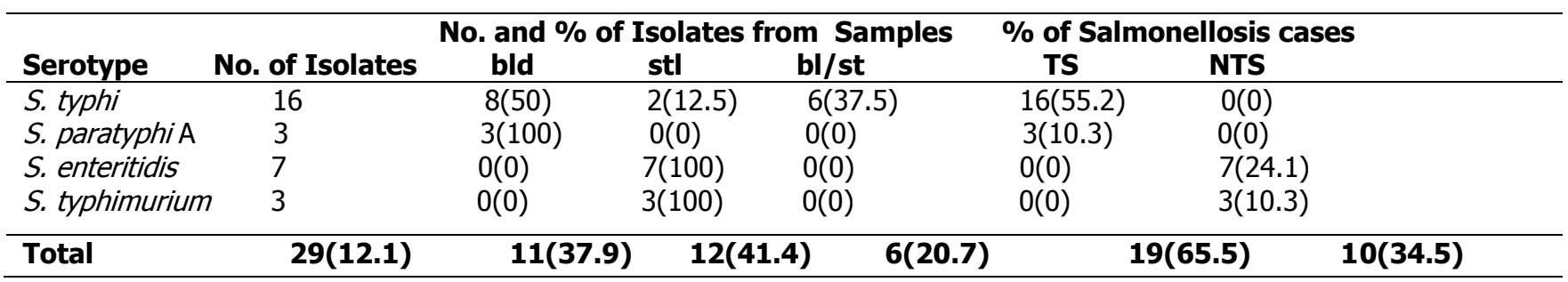

Keys: TS=Typhoidal salmonellosis. NTS= Non-typhoidal salmonellosis. bld= Blood. $\mathbf{s t} \mathbf{l}=$ Stool. $\mathbf{b} \mathbf{l} / \mathbf{s t}=$ Blood$/$ Stool. Figures in parentheses indicate percentages.

Table 2. Distribution of Salmonella Serotypes in relation to Age Groups of patients

\begin{tabular}{|c|c|c|c|c|c|c|c|c|c|}
\hline \multirow{3}{*}{$\begin{array}{l}\text { Age-group } \\
\text { (Yrs) }\end{array}$} & \multicolumn{8}{|c|}{ Salmonella enteritica serotypes } & \multirow[b]{3}{*}{ Total } \\
\hline & \multicolumn{2}{|c|}{ S. typhi } & \multicolumn{2}{|c|}{ S. paratyphi A } & \multicolumn{2}{|c|}{ S. enteritidis } & \multicolumn{2}{|c|}{ S.typhimurim } & \\
\hline & TS & NTS & TS & NTS & TS & NTS & TS & NTS & \\
\hline \multicolumn{10}{|c|}{ CHILDREN } \\
\hline $0-5$ & 4 & 0 & 0 & 0 & 0 & 5 & 0 & 3 & 12(41.4) \\
\hline \multirow{3}{*}{$6-15$} & 7 & 0 & 3 & 0 & 0 & 2 & 0 & 0 & $12(41.4)$ \\
\hline & & & & & & & & Total & $24(82.8)$ \\
\hline & & & & & ADULTS & & & & \\
\hline $16-30$ & 4 & 0 & 0 & 0 & 0 & 0 & 0 & 0 & $4(13.8)$ \\
\hline $31-45$ & 0 & 0 & 0 & 0 & 0 & 0 & 0 & 0 & $O(00)$ \\
\hline$\geq 46$ & 1 & 0 & 0 & 0 & 0 & 0 & 0 & 0 & $1(3.4)$ \\
\hline & & & & & & & & Total & $5(17.2)$ \\
\hline Total & 16 & 0 & 3 & 0 & 0 & 7 & 0 & 3 & $29(12.1)$ \\
\hline
\end{tabular}

Keys: TS= Typhoidal Salmonellosis; NTS= Non-Typhoidal Salmonellosis; Yrs= Years; $\mathbf{n}=$ Number of cases; Figures in parentheses indicate percentages. 


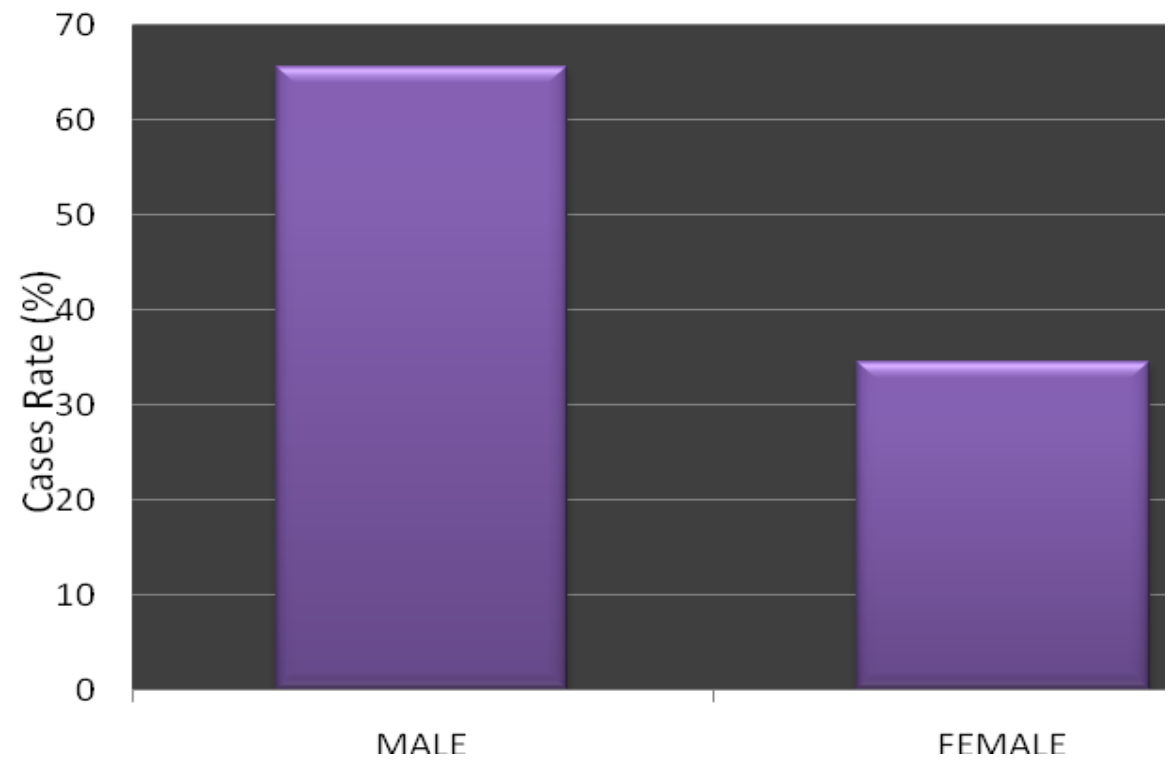

Figure 1. Cases of Salmonellosis with Respect to Sex

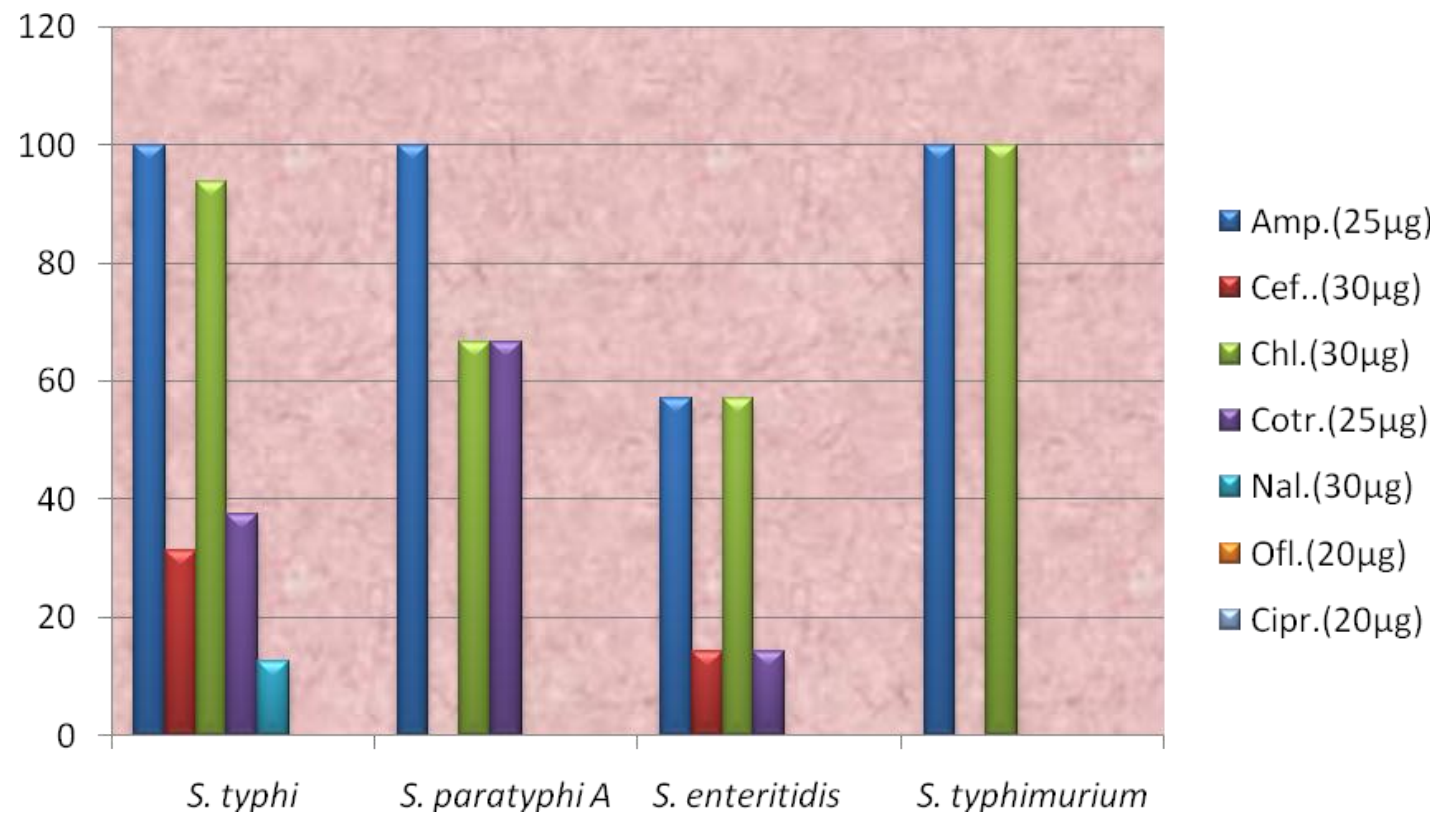

Keys: Amp = Ampicillin. Cef $=$ Cefotaxime. Chl= Chloramphenicol. Cotr $=$ Co-trimoxazol. Nal= Nalizixic Acid. Ofl= Ofloxacin. Cipr= Ciprofloxacin.

Figure 2: Percentages of Antibiotics Resistant Salmonella Serotypes

\section{DISCUSSION}

Twenty nine Salmonella strains, which include $11(37.9 \%)$ from blood and $6(20.7 \%)$ from both blood/stool of patients with pyrexia and 12(41.4\%) from stool samples of patients with gastroenteritis, were isolated in this study, giving an incidence of $12.1 \%$ of Salmonella-associated illness for the period of 8 -months. The results of the study indicated that the frequency of isolation of $S$. tyiphi (55.2\%) from patients with pyrexia was higher than that of $S$. paraityphi $\mathrm{A}(10.3 \%)$. The frequency of isolation of $S$. enteritidis $(24.1 \%)$ from patients with gastroenteritis was higher than that of $S$. typhimurium (10.3\%). Therefore, $S$. typhi was more frequently isolated in typhoidal salmonellosis than $S$. paratyphi A and $S$. enteritidis was more frequently isolated in nontyphoidal salmonellosis than $S$. typhimurium. This finding conformed with the report of Akinyemi et al. (2007) who reported higher frequency of $S$. typhi and $S$. enteritidis in typhoidal and non-typhoidal salmonellosis respectively in Lagos. Mindy, (2003), also reported that paratyphi association in typhoidal salmonellosis is less frequent than $S$. typhi. This may be as a result of the fact that $S$. typhi posseses capsular polysaccharides ( $\mathrm{Vi}$ antigen) (Boyd et al., 1993), which make it to be more virulent than other serotypes implicated in typhoidal- salmonellosis. 
This study also showd that typhoidal salmonellosis $19(65.5 \%)$ cases, are more prevalent than nontyphoidal salmonellosis $10(34.5 \%)$ cases. This is in agreement with the findings of Sood et al., (1999) on "Salmonellosis in developing countries". Threlfall and Ward, (2001), reported decrease in cases of typhoidal salmonellosis in developed countries (due to adequate sanitary measures). However, in these countries, nontyphoidal salmonellosis is more common and most of these cases are associated with food contaminated by Salmonellae (Wright et al, 2007). The differences in the pattern of salmonellosis in developed and developing countries may be as a result of unavailability of portable drinking waters and cultural habits of eating overcooked food in Africa in which Funtua belongs. Out of the 29 cases of salmonellosis in Funtua, children accounted for $24(82.8 \%)$ while adults accounted for 5(17.2\%). This trend appears in agreement with the results of similar studies from Hong Kong(Ling et al., 1991) and Saudi Arabia(Kambal, 1996). However, the present study showed a much higher than usual proportion of isolates from children compared with that of Saudi Arabia where the prevalence is $60 \%$ and $40 \%$ in children and adults respectively (Kambal, 1996). Of the total cases of Salmonellosis, $65.5 \%$ were from male and $34.5 \%$ from female. This is in agreement with report of Kambal (1996) and it may be due to the fact that male (children) are more exposed to infections because they tend to eat more of road side and fast foods compared to their female counterparts who are more reserved and for cultural reason are unexposed to outdoor activities. Most reported cases of Salmonellosis are due to contaminated food and water (Threlfall and Ward, 2001 and Wright et al., 2005).

Resistance to ampicillin, chloramphenicol, cefotaxime and co-trimozaxole was observed in this study which could be as a result of indiscriminate use of these drugs in poultry and humans. The inclusion of preventive doses of antimicrobial agents in poultry feed as growth promoters is often associated with the development of resistance in enteric bacterial flora of poultry. These resistant bacteria contribute to the reservoir of resistant bacteria found in the human intestinal tract including resistant Salmonella (Dupont and Steele, 1987). During this study, $89.7 \%, 82.8 \%$ and $31.0 \%$ of the total Salmonella isolates were resistant to ampicillin, chloramphenicol and cotrimozaxole respectively. This is not surprising because these are most commonly used drugs in human and poultry(Threlfall et al., 2001). The use of antimicrobials for growth-promotion, prophylaxis and treatment of animal's food increases the prevalence of resistance in human pathogens, particularly non-

\section{REFERENCES}

Akinyemi, K. O., Babajide, S. B. And Akintoye, O. C.(2007): Salmonella in Lagos, Nigeria: Incidence of Plasmodium falciparumassociated co-Reduced Susceptibility to Fluoroquinolones. Health Popul Nutr 3: 351358. typhoidal salmonellosis (Singer et al., 2003). Out of the 29 isolates, 6(20.7\%) were resistant to cefotaxime. This is in contrary to reports by Campos et al. (1990) and, Sonstein and Burnham (1993) where high percentage of Salmonella isolates were sensitive to cefotaxime. This may be as a result of indiscriminate use of this antibiotics. This trend is of particular concern because the extended spectrum cephalosporins are the antibiotics of choice for children(Weill et al., 2004). High susceptibility of Salmonella spp. was observed against fluoroquinolones (ofloxacin and ciprofloxacin). Despite the fact that $2(6.9 \%)$ of the isolates were resistant to nalidixic acid, $100 \%$ of them were sensitive to ofloxacin and ciprofloxacin. This finding is similar to a report by Campos et al. (1990) and Sonstein and Burnham (1993). However , in Lagos, Nigeria, Akinyemi et al. (2007) reported $18 \%$ reduced susceptibility of Salmonella spp. to ofloxacin and ciprofloxacin. The high susceptibility of Salmonella spp. to fluoroquinolones recorded in this study may be connected to relatively expensive cost of ciprofloxacin and ofloxacin. Therefore, fluoroquinolones are not used indiscriminately because not many could afford them.

This is the first study on antimicrobial susceptibility pattern of Salmonella species in Funtua, Nigeria (to the best of our knowledge). There was no observation of reduced susceptibility of these organisms to fluoroquinolones compared to Asia and United Kingdom and some other Europeans countries where resistance to fluoroquinolones have been recorded most likely due to approval of these drugs for use in animals (WHO, 1998). Though there was report of reduced susceptibility of Salmonella spp. to fluoroquinolones in Lagos, by Akinyemi et al. (2007), differences in drug use patterns in the two geographically distinct areas may account for the diffrences in the observed susceptibility patterns.

\section{CONCLUSION}

In conclusion, majority of the Salmonella isolated were from children with $S$. typhi accounting for the highest percentage of the isolates.

\section{Recommendations}

More importantly, there is need for the Government to enforce the existing laws that prohibit smuggling of all kinds of animal products and the sale of drugs by unauthorized people.

\section{Acknowledgment}

The authors acknowledge and appreciate the moral and financial support given by Umaru Musa Yar'adua University, Katsina.

Akinyemi, K. O., Coker A. O., Olukoya, D. K., Oyefolu, A. O., Amoroghoye, E. P. and Omonighehin, E. O. (2000): Prevalence of multi-drug resistant Salmonella typhi among clinically diagnosed typhoid fever in Lagos, Nigeria. $Z$ Naturforsch:_55: 489-93. 
Barrow, G. F. And Feltham R. K. A. (1993): Cowans and Steel's Manual for the Identification of Medical Bacteria, $3^{\text {rd }}$ ed. Editors: Cambridge University Press, pp. 331

Boyd, E. F., Wang, F. S., Beltran, P., Plock, S. A., Nelson, K. and Selander, R. K. (1993): Salmonella Reference Collection B (SARB); Strains of 37 Serovars of Subspecies. J. Gen. Microbiol._139: 1125-1132.

Campos L. C., Reis, E. M., Ribeiro and Dias, J. C. (1990): In vitro Susceptibility of Salmonella to Ciprofloxacin and Pefloxacin Compared With Three other Antibiotics. Chemotherapy: 36 (1): 29-32

Centre for Disease Control and Prevention (CDC) (2009):

http:/www.cdc.gov/nczned/dfbmd/disease listening/salmonellosis (4/24/2009)

Dupont, H. L. and Steele, J. H. (1987). Use of antimicrobial agents in animal feeds. Implication for human health. Rev. Infect. Dis: 9:447.

Giannella, R. (1996): Salmonella. In Baron, S. Editor. Medical Microbiology. $4^{\text {th }}$ ed. New York: Churchill Livingstone. Available at: http://www.gsbs.utmb.edu/microbook/ch021 .htm.

Guithrie, R. K. (1991): Control of Salmonella Spread. In: Saltionella pp. 63-81. CRC press, Boca Raton, FL.

Hakanen, A., Kotilainen, P., Helenius, H. and Siitonen A. (2001): Reduced Fluoroquinolones Susceptibility in Salmonella enteritica Isolates In Travellers Returning From Southeast Asia. Emerg infect Dis. 7: 996-1003.

Kambal, A.M. (1996): Antimicrobial susceptability and serogroups of Salmonella isolates from Riyadh, Saudi Arabia. Int J Antimicro Agent. 7:265-9

Ling, J. M, Zhou G.M, Woo T. H. S and French G. H.(1991): Antimicrobial susceptibility and $\beta$ lactamase production of Hong Kong isolates of gastroenteric Salmonellae and Salmonella typhi. J Antimicrob Chemother 28:877-85.

Lin-Hui, S., Cheng-Hsun, C., Chishih, C. and Jonathan, T. O. (2004): Antimicrobial Resistance In Non-Typhoidal Salmonella Serotypes. A global challenge. Clin. Infectious Disease. 39: 546-51.

McClelland, M., Porwollik, S., Boyd, E. F., Choy, C., Cheng, P., Florea, L. and Proctor, E. (2004): Characterization of Salmonella enteritica subspecies I serovars by use of Microarrays. Journal of America Society of Microbiology pp. 5885-5898, 186, No.17.

Mindy, J. P. (2003): Manual for the Laboratory Identification and Antimicrobial Susceptibility Testing of Bacterial Pathogens of Public Health Importance in The Developing World. Pp.103-120

Porwollik, S., Frye, J., Florea, L. D., Blackmer, F., and McClelland, M. (2003): A non redundant microarray of genes for two related bacteria Nucleic Acid Res. 31: 1869-1876.
Rusin, P., Enriquez, C., Jonson, D., and Yerba, C. P. (2002): Environmentally Transmitted Pathogens. In: Maier R. M., Pepper I. L., Yerba C. P. (Eds), Environmental Microbiology. Academic press, San Diego. pp 447-487.

Singer, R. S., Finch, R., Wegener H. C., Bywater R., Walters J. and Lipsitch, M. (2003): Antibiotic Resistance; The Interplay Between Antibiotic Use in Animals and Human Beings. Lancet. Infect. Dis: 3:47-51.

Sonstein, S. A. and Burnham, J. C. (1993): Effect Of Low Concentration Of Quinolones Antibiotics On Bacterial Virulence Mechanisms. Diagn. Microbial. Infect. Dis: 16 (4): 277-289.

Sood, S., Kapil, D., Das, B., Jain, Y. and Kabra, S. K. (1999): Re-emergence of Chloramphenicol sensitive Salmonella Typhi. Lancet: 353: 1241-2

Threlfall, E. J. and Ward, L. R. (2001): Decreased Susceptibility to Ciprofloxacin in Salmonella enteritica serotype Typhi, United Kingdom. Emerg Infect Dis: 7: 448-50

Weill, F. X., Demartin, M., Tande, D., Espie, E., Rakotoarivony, I. and Grimont, P. A. (2004): SHV-12 Like Extended-Spectrum- $\beta$ Lactamase-Producing Strains Of Salmonella enteric serotypes Babelsberg and Enteritidis Isolated In France Among Infants Adopted From Mali. J Clin Microbial: 42: 2432-7.

WHO. (1998): Use Of Quinolones In Food Animals And Potential Impact On Human Health; Report and Proceeding Of WHO Meeting, Geneva, Switzerland, 2-5 June 1998. Geneva: Division Of Emerging And Other Communicable Diseases Surveillance And Control, World Health Organization. pp 130. (WHO/EMC/ZD/98.12).

WHO. (2010). Laboratory Protocol: "Susceptibility testing of Enterobacteriaceae using disk diffusion". WHO Global Foodborne Infection Network.

Wrigth, J. G., Tengelsen, L. A., Smith, K. E. J.B., Frank, R. K. and Gredon, J. H. (2005): Multidrug resistance Salmonella Typhimurium in four animal facilities. Emerg Infect Dis: 11: $1235-4$ 